\title{
Arylpropionic Alcohols via Enzyme-Mediated Dynamic Kinetic Resolution
}

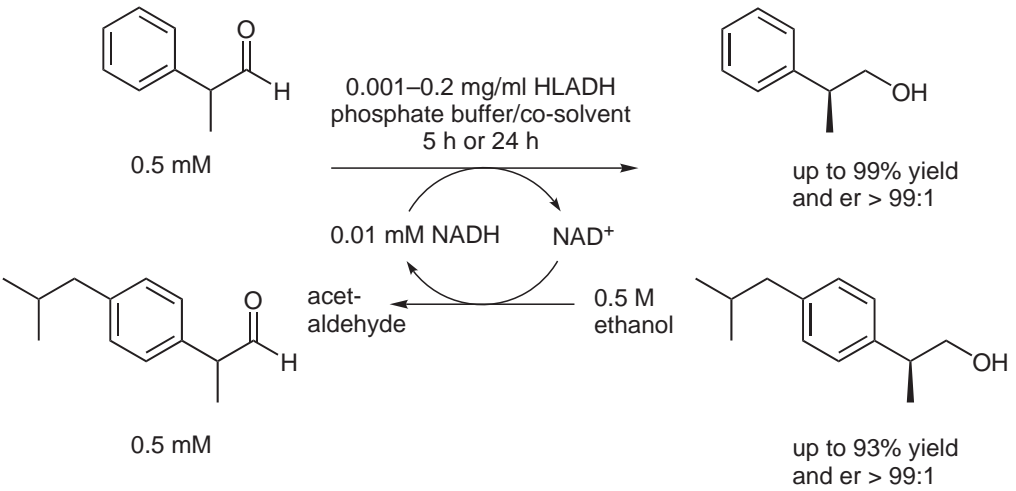

Significance: The authors report their preliminary studies on the reduction of two 2-arylpropionic aldehydes to enantioenriched alcohols via a dynamic kinetic resolution. The enzyme catalyst is commercially available horse liver alcohol dehydrogenase (HLADH), with NADH as a cofactor. Since the enzyme regenerates its cofactor in the presence of ethanol, substoichiometric amounts of $\mathrm{NADH}$ are sufficient. It was found that organic cosolvents tetrahydrofurane and acetonitrile, which increase the solubility of the starting material, are tolerated in up to $10 \mathrm{vol} \%$ with respect to the buffer solution. With a change in the protocol, almost pure hexane (up to 99 vol\%) can also be tolerated as solvent.
Comment: The dynamic kinetic resolution is a powerful tool to convert a starting material into an enantioenriched or even enantiopure product, theoretically in $100 \%$ yield, which is a distinct advantage over kinetic resolution methods. The catalyst in this reaction proves to be 'multitasking': Not only does it perform the reduction of the aldehyde, but there is also no need for adding a special racemization catalyst, and it recycles NADH by oxidizing ethanol and reducing $\mathrm{NAD}^{+}$. This greatly lowers the amount of expensive NADH employed in the reaction.
Key words

enzymes

dynamic kinetic resolution

alcohol dehydrogenase

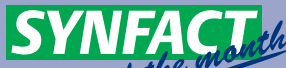

